\title{
Automatic Detection and Localization of Macular Edema
}

\author{
S. Sumithra \\ M. Tech scholar \\ Department of ECE \\ JNTUACE Ananthapuramu \\ Andhra Pradesh, India
}

\author{
K. R. Remya \\ Research Scholar \\ Department of ECE \\ JNTUACE Ananthapuramu \\ Andhra Pradesh, India
}

\author{
Dr. M. N. Giri Prasad \\ Professor \\ Department of ECE \\ JNTUACE Ananthapuramu \\ Andhra Pradesh, India
}

\begin{abstract}
Diabetic retinopathy is an eye disease and causes vision loss to the people who are suffering longer from the diabetes. Exudates, bright and red lesions are identified in the diabetic retinal eye. Automatic detection and localization of macular edema is a challenging issue since exudates have non uniform illumination and are low contrasted. Proposed algorithm to detect macular edema encompasses Simple Linear Iterative Clustering, Fisher linear discriminant and Support vector machine classifer. Optic Disc extraction prior to exudates extraction is also introduced. Performance of the proposed detection algorithm is tested on easily available databases: Diaretdb1, Messidor and E_optha Ex. Proposed method shows an accuracy of $\mathbf{9 7 . 8 1 \%}$, specificity 98.65 and Sensitivity 82.71\%.
\end{abstract}

Keywords:- Macular Edema, Exudates, Superpixel, Simple Linear Iterative Clustering, Fisher's Linear Discriminant, Support Vector Machine.

\section{INTRODUCTION}

Diabetes is a challenging issue with estimated $8.7 \%$ diabetic population between 25 and 70 years in India. World Health Organization says that 96000 thousand people in India are suffering from type 2 diabetics by the end of 2030.Diabetic retinopathy is a critical stage of Diabetes Mellitus (DM), leads to the cause of blindness who are suffering longer from the Diabetes[1]. Overall 30\% of the diabetic patients from the world have diabetic retinopathy. Non-proliferative and proliferative are two types of stages in DR[2],[3].Non-proliferative causes damage gradually and proliferative diabetic retinopathy causes abrupt damage to the vision and introduces abnormal blood vessels and formation of new vessels in the retina. Blurred vision and patches in the retina are the signs of diabetic retinopathy.

Paper [4];Computational intelligence technique is used for extraction of exudates. Fuzzy c means clustering algorithm is used to segment the image with the features like size, edge strength and texture. Before segmenting the image some preprocessing steps are used like color normalization and contrast enhancement [5].

Paper [6]; Superpixel segmentation method is used for image segmentation and a supervised classification method to classify the exudates from non-exudates, is used.
Paper [7]; mainly focus on the detection of exudates (mass of cell and fluid seeped out of damaged blood vessel in the macula region). Partial Least Squares method is used for classification.

Figure 1 shows the corresponding retinal funds images with (a) macular edema (exudates in macula region) (b) Exudates shown by arrows.

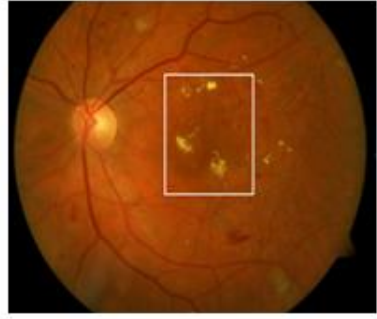

(a)

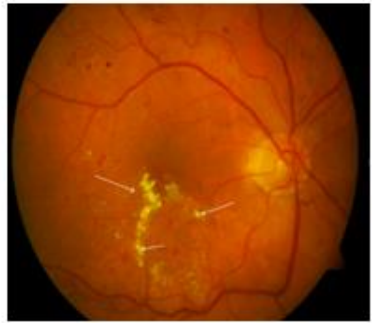

(b)
Fig 1:- Fundus images having exudates in macula region

\section{IMPLIMENTING WORK}

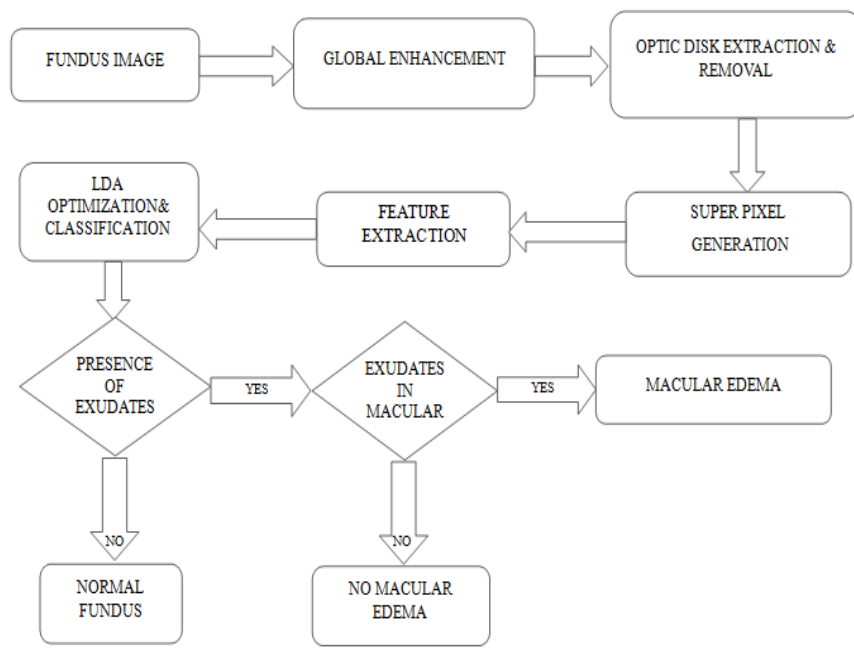

Fig 2:- Block diagram of the proposed algorithm

A five step process is undergone in this paper to detect exudates and macular edema. In the first step funds images are enhanced using global enhancement technique. This is used to enhance the quality of the image so that presence of the small exudates and macula region is highlighted very clearly. Next optic disc is localized using an algorithm 
which detects the brightest area of funds image. Localized optic disc is removed by Morphological operation. Next the enhanced retinal image is clustered into superpixels (group of pixels with similar characteristics) using Simple Linear Iterative Clustering (SLIC)algorithm[8]. Segmentation is done by color based clustering. In the next step features are extracted and a supervised classification algorithm called Linear Discrimination Analysis (LDA) [9] is used to distingue the true exudates from spurious exudates. In the final step macula region and presence of exudates in macula region is also identified. Figure 2 represents the flowchart of implementing work of the automatic detection and localization of exudates in macula region.

\section{PREPROCESSING}

To identify the true exudates, color normalization and contrast enhancement techniques are essential. Color digital funds images often have non-uniform illumination. Moreover, retinal images are naturally low in contrast. The contrast of funds images reduces, as it moves from optic disc. With these characteristics, detection of exudates will be difficult. So, we use Global enhancement method for checking contrast variability and brightness within the retinal image by transforming RGB into YIQ [10]. To yield a set of new (Rmod, Gmod, Bmod) component as shown below we applied a transformation technique.

$$
(R, G, B) \rightarrow(Y, I, Q)
$$

Transforming the RGB color channels into YIQ color model and processing the luminance channel of YIQ model with optimal weights [11].

$$
Y \_ \text {channel }=a^{*} Y+b^{*} Y+c^{*} Y
$$

then transformed to

$$
\left(Y_{\_} \text {channel }, I, Q\right) \rightarrow(R, G, B)
$$

Where the parameters $\mathrm{a}, \mathrm{b}$ and $\mathrm{c}$ depend on the characteristics of the images. These parameters are used to reduce the local luminance variability $\sigma_{\mu}$ throughout channel and enhance the mean contrast levels $\mu_{\sigma}$ and $\mu_{c}$ within the channel.

Figure 3 represents the (a) Original image and (b) shows the enhanced image

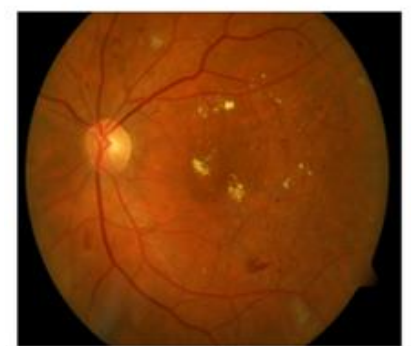

(a)

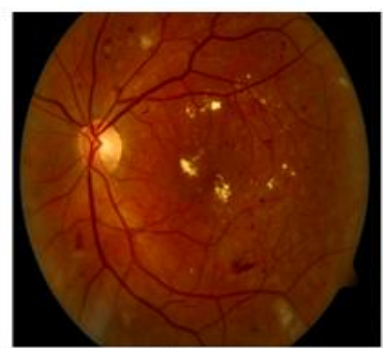

(b)
Fig 3:- Preprocessing of funds images
Table 1 represents the measure of enhancement of the original and preprocessed image for different images.

\begin{tabular}{|c|c|c|}
\hline \multicolumn{3}{|c|}{ Measure of Enhancement } \\
\hline Image & $\begin{array}{c}\text { Before } \\
\text { enhancement }\end{array}$ & $\begin{array}{c}\text { After } \\
\text { enhancement }\end{array}$ \\
\hline Image015 & 5.3821 & 5.8587 \\
\hline Image006 & 1.6771 & 3.1005 \\
\hline Image019 & 4.2717 & 5.8020 \\
\hline Image005 & 4.2687 & 5.3821 \\
\hline
\end{tabular}

Table 1:- EME of the retinal funds images

\section{DISC EXTRACTION}

Optic disc (OD) is significant source of true positives in exudates detection since the exudates have same intensity as OD and it may have the chance to detect as exudates. Hence the chance of miss classification is more. To avoid the problem of miss classification, OD is detected and removed. It has two steps as follows:

Green channel extraction: Retinal images are usually low in contrast, but exudates are clearly visible in green channel due to high contrast. Hence green channel extracted.

Detection and Extraction of Optic disc:Filtering and morphological operations are performed on the green channel image. Median filtering is used to eliminate the noise from the image and contrast stretching technique is adapted as a morphological operation to enhance the range of brightness values present on an image. This technique is performed by applying the threshold as Thre $=\left(\mathrm{I}_{\mathrm{cs}}-10\right)$ on the contrasted image $\left(\mathrm{I}_{\mathrm{cs}}\right)$, and output binary image obtained as follows

$I_{b w}(x, y)=\left\{\begin{array}{lll}1, & \text { if } & I_{b w}(x, y)>\text { Thre } \\ 0 & \text { else }\end{array}\right\}$

\section{SUPERPIXEL GENERATION}

Simple Linear Iterative Clustering is the advanced kmeans clustering algorithm used to segment the image into series of super pixel. It is simple and efficient method to decompose image into homogenous regions.

$$
\psi(x, y)=\left[\begin{array}{l}
\lambda x \\
\lambda y \\
I(x, y)
\end{array}\right]
$$

Where $\lambda$ balances the spatial and appearance of the element vectors, forcing a level of spatial regularization to the separated areas. In SLIC, the accompanying two parameters, the region size and the regularizer are used. First the image is segmented into a cluster with step region size. Center of the cluster is used to initialize a corresponding k-means. Finally, the clusters and k-means centers are recreated by using Lloyd algorithm, yielding the segmented image as result. For further confinement and 
improvement, during the merging of k-means clusters every pixel will be allocated to just the $2 * 2$ focuses relating to lattice tiles adjoining the pixel. The parameter "regularizer" has a trade-off between spatial regularization and clustering appearance.

$$
\lambda=\frac{\text { regularizer }}{\text { regionsize }}
$$

SLIC [12]algorithm begins by splitting the image into regular $M \times N$ tiles, where $\mathrm{M}$ and $\mathrm{N}$ are

$$
M=\frac{\text { imagewidth }}{\text { regionsize }} \quad N=\frac{\text { imageheight }}{\text { regionsize }}
$$

Super pixels or k-means cluster is initialized from its center

$x_{i}=$ round $_{i} \frac{\text { imagewidth }}{\text { regionsize }} y_{j}=$ round $_{j} \frac{\text { imagewidth }}{\text { regionsize }}$

Minimize the edge strength to $3 \times 3$ neighborhoods to avoid placing these centers over picture discontinuities

$$
\begin{aligned}
& \operatorname{edge}(x, y)=\|I(x+1, y)-I(x-1, y)\|_{2}^{2}+ \\
& \|I(x, y+1)-I(x, y-1)\|_{2}^{2}
\end{aligned}
$$

From the K-means clustering algorithm the regions are obtained, started from the centers

$$
c=\{\psi(x i, y j), i=0,1, \ldots, M-1 j=0,1, \ldots N-1\}
$$

In case any segment's area is lesser than the threshold minimum region, then SLIC combines the segment into larger ones.

\section{FEATURE EXTRACTION}

The extracted channels of RGB image views that exudates have multiple background contrast in various. Means is the normalized feature of saturation channel and it can be normalized by dividing it with global mean. Standard deviation and global mean of each preprocessed green channel retinal image are also included into sampled feature set named as Mean and STD.

\section{A. Features}

The aim of the feature extraction stage is pixel characterization by means of a feature vector, a pixel representation in terms of some quantifiable measurements which may be easily used in the classification stage to decide whether pixels belong to a exudates or not. In this paper, the following sets of features were selected Gray level co-occurrence matrix (GLCM), GLCM is considered to extract around 20 texture features In addition to abovementioned features, Shannon, Renyi, and Kapur entropies were also computed from green channel image

$$
f_{i}^{\prime}=\frac{f_{i}-\mu_{i}}{\sigma_{i}}
$$

Where $\mu_{i}$ is the mean of the $i_{\text {th }}$ feature, and $\sigma_{i}$ is its standard deviation.

Contextual feature is also extracted from the preprocessed green channel image and is indicated by $S_{i}$ as follows:

$$
\begin{aligned}
& S_{i}=\frac{1}{N_{i}} \frac{\text { Mean } \_C_{i}}{\text { Mean }} \sum_{j \in N(i)} \frac{d_{j}}{D_{j}} \\
& d_{j}=\text { Mean }_{-} C_{i}-\text { Mean } \_C_{j} \\
& D_{j}=\sqrt{\left(\left\|p_{i}-p_{j}\right\|_{2}^{2}\right)}
\end{aligned}
$$

Where $\mathrm{j} \in \mathrm{N}$ (i), if there are $\mathrm{p}$ closely packed candidate regions $C_{i}$ where $i=1,2 \ldots p$, then $d_{j}$ is the measurable brightness and $D_{j}$ is the spatial distance between candidate region $C_{i}$ and its neighbor candidate region $C_{j}$. In order to differentiate exudates with other luminous regions, the gray distance $d$ between neighboring superpixels is considered. If Sum of the weighted gray distance is more than other bright region then it is treated as exudates.

\section{CLASSIFIER}

The proposed work implements two numbers of the commonly used two-class classifier systems, which helps to classify the given retinal pictures into the normal/DR class. Extracted features are given to the classifiers.

\section{A. Support Vector machine classifier:}

The support vector machine (SVM) is superior of all machine learning algorithms. SVM employs optimization algorithms to locate the optimal boundaries between classes. The optimal boundaries should be generalized to unseen samples with least errors among all possible boundaries separating the classes, therefore minimizing the confusing between classes. There are number of kernels that can be used in Support Vector Machines models. These include linear, polynomial, radial basis function (RBF) and sigmoid

SVM technique is most useful for data classification. In this technique, data divided into sets called training, testing involving classification task. Training set contain multiple features. SVM model able to predict the target values of tested samples when characteristics of test data given as input. It distinguish the problem based on supervised learning algorithm from the given labeled data. A Support vector Machine is a potentate classifier without ambiguity by a sovereign hyper plane. The data which is to be classify into classes $[+1,-1]$, can separate by a hyper plane which is denoted by $\mathrm{Wx}+\mathrm{b}=0$. The values of $\mathrm{W}$ and $\mathrm{b}$ can determine by solving the constrained minimization problem. SVM do the classification based on matching the inputs and the trained features. SVMSTRUCT is created to 
classify the test image and yields the predicted class group. The obtained result assigned to the variable.

\section{B. Fisher Discrimination Analysis (FDA):}

To distinguish between exudates and non exudates we use Fisher Discrimination Analysis (FDA) classifiers [13]. In this analysis, the function called discriminant that takes an input $\mathrm{x}$ as a vector and allocates it to one of the $\mathrm{k}$ classes, denoted by $c_{k}$. The most effective representation of a linear discriminate function is received by means of taking a linear function of the input vector so that

$$
y(x)=w^{T} x+w_{o}
$$

Where $\mathrm{w}$ is the weight vector and $\mathrm{w}_{0}$ is threshold. Parameters an input vector $\mathrm{x}$ is assigned to $\mathrm{c}_{1}$ if $\mathrm{y}(\mathrm{x})>0$ and to $\mathrm{c}_{2}$ if $\mathrm{y}(\mathrm{x})<0$ where $\mathrm{c}$ is a class and $\mathrm{y}(\mathrm{x})=0$ is decision boundary, inside D-dimensional input space, which corresponds to D-1 dimensional hyper plane.

Fisher Discriminate: It is a dimensionality reduction technique. Suppose an input vector $\mathrm{x}$ with $\mathrm{D}$-dimensional then it projected down to single dimension by

$$
y(x)=w^{T} x
$$

Consider two class classification problems: There are $\mathrm{N}_{1}$ exudates of class $\mathrm{c} 1$ and $\mathrm{N}_{2}$ non-exudates of class $\mathrm{c} 2$, mean vectors of the two classes are

$$
m_{1}=\frac{1}{N_{1}} \sum_{n \in c_{1}} x_{n} \quad m_{2}=\frac{1}{N_{2}} \sum_{n \in c_{2}} x_{n}
$$

The separation value of the classes is the separation of the projected class means. Choose $\mathrm{w}$ so as to maximize the projected class mean.

$$
\begin{aligned}
& m_{2}-m_{1}=w^{T}\left(m_{2}-m_{1}\right) \\
& \text { where } \quad m_{k}=w^{T} m_{k}
\end{aligned}
$$

$m_{k}$ represents the mean of intended data from $c_{\mathrm{k}}$. This separation feasibly produces abruptly large simply by raising the weight of $\mathrm{w} . \quad \sum_{i} w_{i}^{2}=1$.

The reasonable loss of information and problem of overlapping occurs with the projection of classes that are well distributed in theoriginal2-Dspace $(\mathrm{x} 1, \mathrm{x} 2)$ at the moment of projection onto the line joining of their means.

$$
y(x)=w^{T} x \text { is the projection formula that converts }
$$
the set of labeled data points of $x$ to a labeled set in the $y$ dimensional space. Within class covariance of the transformed sample set from class $\mathrm{c}_{\mathrm{k}}$ is obtained from $s_{k}^{2}=\sum_{n \in c_{k}}\left(y_{n}-m_{k}\right)^{2} \quad$ where $\quad y_{n}=w^{T} x_{n}$

$s_{1}^{2}+s_{2}^{2}$ is the total within class variance of whole data. The relation between within class variance and between class variance is given by the fisher's analysis

$$
J(w)=\frac{\left(m_{2}-m_{1}\right)^{2}}{s_{1}^{2}+s_{2}^{2}}
$$

Using the eq. (13), (16), and (18) rewrite eq. (19) as

$$
J(w)=\frac{w^{T} s_{B} w}{w^{T} s_{w} w}
$$

Where between class covariance matrix is marked by $\mathrm{SB}_{\mathrm{B}}$

$$
s_{B}=\left(m_{2}-m_{1}\right)\left(m_{2}-m_{1}\right)^{T}
$$

And within class covariance matrix is marked by $\mathrm{Sw}_{\mathrm{W}}$ $s_{W}=\sum_{n \in c_{1}}\left(x_{n}-m_{1}\right)\left(x_{2}-m_{1}\right)^{T}+\sum_{n \in c_{2}}\left(x_{n}-m_{2}\right)\left(x_{n}-m_{2}\right)^{T}$

Differentiating the eq. (22) with $\mathrm{w}$, we find that $\mathrm{J}(\mathrm{w})$ is optimized if

$$
\left(w^{T} s_{B} w\right) s_{W} w=\left(w^{T} s_{W} w\right) s_{B} w
$$

eq. (21), states that $\mathrm{s}_{\mathrm{B}}$ follows the direction of $\left(\mathrm{m}_{2}-\mathrm{m}_{1}\right)$. From this we obtain

$$
w \quad \alpha \quad s_{w}^{-1}\left(m_{2}-m_{1}\right) .
$$

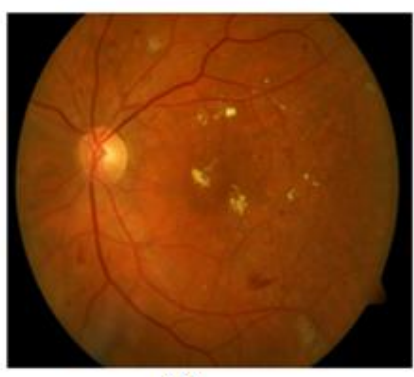

(a)

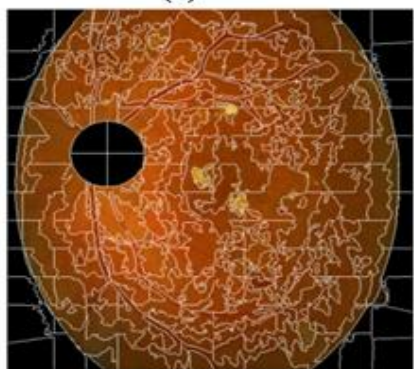

(c)

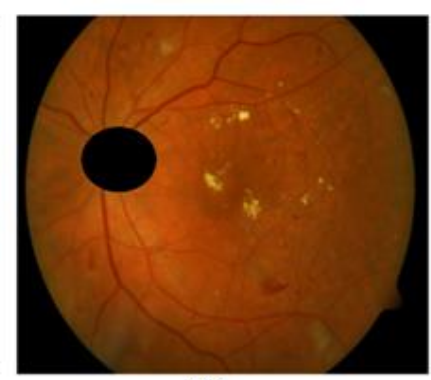

(b)

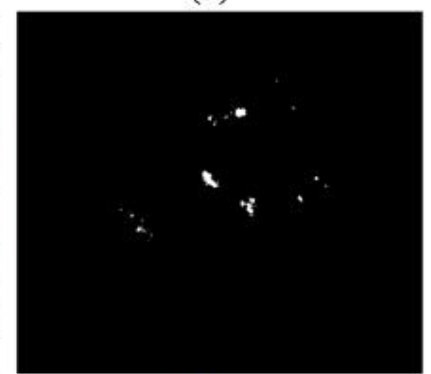

(d)
Fig 4:- Steps carried out to perform extraction of exudates(a) Fundus image with exudates. (b) Extracted optic disc. (c) Generated superpixels (d) Detected Exudates

Figure 4 represents the outputs of the methods of optic disc extraction, superpixels segmentation and exudates detection 


\section{DETECTION AND LOCALIZATION OF MACULAR EDEMA}

The macula is an oval-formed pigmented region close to the focal point of the retina. Presence of exudates in the region of Diabetic macular edema (DME)[14] is one of the extreme entanglements of diabetic retinopathy causing serious vision misfortune and prompts visual impairment in extreme cases whenever left untreated. The macula region is localized by the simple method of identifying zero intensity values of all color channels in retinal funds image.

\section{A. Algorithm to Detect Macular Edema:}

$>$ Preprocess the image to view the particulars clearly.

$>$ Find the minimum intensity value of the pixels in all the channels localizing region by region growing process in the image and identified as macula region.

Then by making the threshold automatically in the macula region exudates can be detected and counted by making the initial count of exudates in the macula region zero.

$$
\begin{aligned}
& \text { Count }=0 ; \\
& \text { for } i=m ; \\
& \qquad \text { for } j=n ;
\end{aligned}
$$

Count $=$ count +1 ;

$$
\text { if (image }(i, j)>=T)
$$

$$
\text { else }
$$$$
\text { end }
$$

end

The count indicates the number of exudates in the macula region.

Dividing the image macula region into different zones then severity of the decease is determined by the exudates in zones.

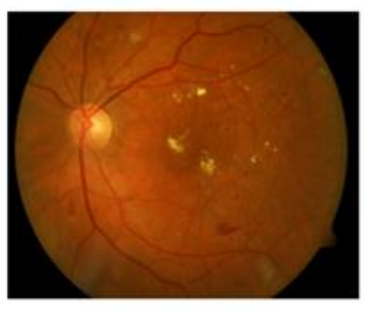

(a)

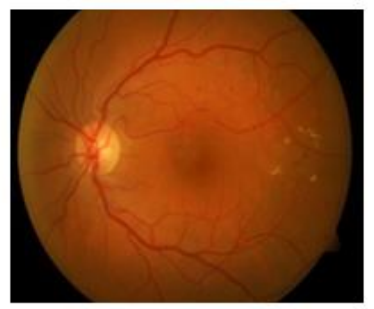

(c)

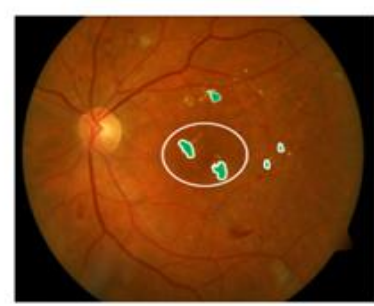

(b)

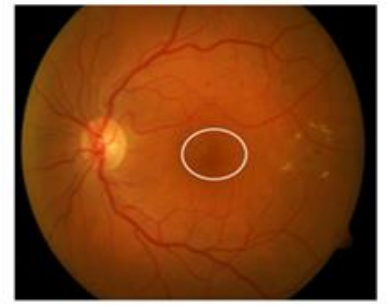

(d)
Fig 5:- Detection and localization of macular edema

Figure 5 shows (a) Fundus image with macular Edema (b) Presence of Macular Edema detected (c) Fundus Image without macular edema (d) Detected clear Macular region

\section{RESULT AND PERFORMANCE MEASURE}

Three easily available databases are used for this work that are Diaretdb1[15], E-ophtha_EX[16] and Messidor[17].

\begin{tabular}{|c|c|c|c|c|}
\hline Database & $\begin{array}{c}\text { Abnormal } \\
\text { Images }\end{array}$ & $\begin{array}{c}\text { Normal } \\
\text { Images }\end{array}$ & Total & Resolution \\
\hline Diaretdb1 & 84 & 5 & 89 & $\begin{array}{c}1152 \times \\
4500\end{array}$ \\
\hline E-Ophtha & 47 & 35 & 82 & $\begin{array}{c}1440 \times 960 \\
2544 \times 696\end{array}$ \\
EX & & 546 & 1200 & $\begin{array}{c}1440 \times 960 \\
240 \times 488 \\
2304 \times \\
\text { Messidor }\end{array}$ \\
& 654 & & & 1536 \\
& & & & \\
\hline
\end{tabular}

Table 2:- Summary of Database Used

\section{A. Performance Measure:}

The performance of exudates detection is done by measuring the three different kinds of performance measures such as sensitivity, specificity and accuracy. The capability of identifying the patient cases correctly is called sensitivity and is defined by the following equation

$$
\text { sensitivity }=\frac{T P}{T P+F N}
$$

The capability of identifying healthy cases correctly is called specificity

$$
\text { Specificity }=\frac{T N}{T N+F P}
$$

The capability of identifying the patients and healthy cases correctly is called accuracy.

$$
\text { Accuracy }=\frac{T P+T N}{T P+T N+F N+F P}
$$

True positive (TP) indicates the number of exudates identified correctly. True negative (TN) indicates the number of spurious exudates identified correctly. FP is false positive indicates the number of spurious exudates identified as exudates where $\mathrm{FN}$ is false negative indicate the number of true exudates identified as spurious exudates. Table 3 represents the sensitivity specificity and accuracy of for different images taken from Diaretdb1 database. 


\begin{tabular}{|c|c|c|c|}
\hline Image & Sensitivity & Specificity & Accuracy \\
\hline image005 & 0.8877 & 0.9485 & 0.9481 \\
\hline image013 & 0.8937 & 0.9842 & 0.9768 \\
\hline image016 & 0.8881 & 0.9891 & 0.9741 \\
\hline image014 & 0.8899 & 0.9 & 0.9749 \\
\hline image019 & 0.8972 & 0.9644 & 0.9576 \\
\hline image0015 & 0.8950 & 0.9570 & 0.9560 \\
\hline image034 & 0.8961 & 0.9768 & 0.9781 \\
\hline image025 & 0.8689 & 0.9759 & 0.9826 \\
\hline image048 & 0.8732 & 0.9689 & 0.9790 \\
\hline average & 0.8271 & 0.9865 & 0.9781 \\
\hline
\end{tabular}

Table 3:- Performance evaluation of exudates detection

\section{B. Receiver Operating Characteristics:}

It is the graph represents the diagnostic ability of a classifier system. It measures the performance of the system Figure 6 shows the graphical representation of ROC curve with FPR on X-axis and TPR on Y-axis.

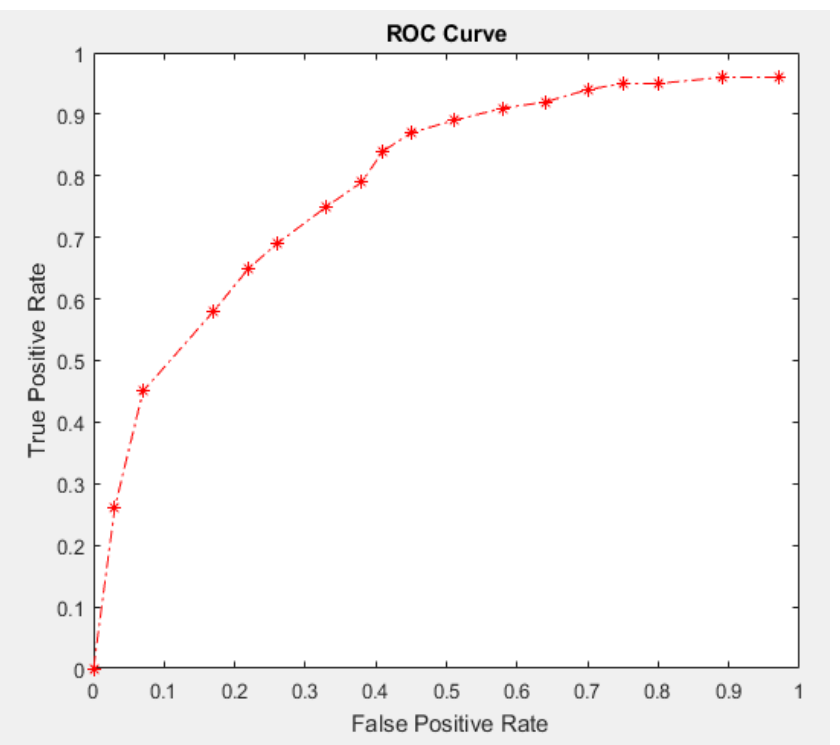

Fig 6:- ROC graph

\section{CONCLUSION}

This paper presented a method that would be used in the detection of Macular edema using different image processing techniques. OD extraction and its removal from the preprocessed image will aid in efficient detection of exudates. Since the later has the same intensity as that of exudates. Detection and removal of exudates encompasses SLIC and fisher linear discriminant analysis. The Method has been performed on three data bases Diaretdb1, E_optha Ex and Messidor database and shows an accuracy of $97.84 \%$, specificity $98.67 \%$ and Sensitivity $88.79 \%$.

\section{REFERENCES}

[1]. D. Welfer, Segio B Junior. Automatic Detection of Heamorrhages And Microanuearysms in Color Fundus Images. Southeast Asian J Trop Med Public Heal. 2003; 34: 751-757.

[2]. S Mashohor, Z. A Omar, F.N.M. Mahfudz, M. Hanafi , Muna. im, M. Automatic diabetic retinopathy classification and detection system. 7th IEEE International Conference on System Engineering and Technology (ICSET). 2017; 162-165.

[3]. Nourreddine, B., Mohamed, B., Abdelmalek, B. Yazid, C, Assia, C. Non-proliferative diabetic retinopathy(NPDR) detection based on mathematical morphology. IEEE 4th Middle East Conference on BiomedicalEngineering (MECBME). 2018; 219-222.

[4]. R. Markham, A. Osareh, and B. Shadgar. An Approch Based On Computational-Intelligence for Detection of Exudates in Diabetic Retinopathy Retinal Images. IEEE Transactions on Information Technology in Biomedicine. 2009; 13: 535-545.

[5]. Ruggeri A., Foracchia .M, and Grisan .E. Normalization of Luminosity and contrast in retinal fundus images. Med. Image Anal. 2005; 9: 179-190.

[6]. Yi, Y., \& Du, W., Zhou, W., Wu, C. Exudates Detection in Color Fundus Images Using Superpixel Multi-Feature Classification Automatically. 2017; 17077-17088.

[7]. M Pattichis, Agurto, C. S Nemeth, P Soliz, Murray, V., Yu, H., Wigdahl, J. Detection of Exudates in the Macula Using Multiscale Optimization Method. IEEE Journal of Biomedical and Health Informatics. 2014; 18: $1328-1336$.

[8]. K Smith, S. Süsstrunk, R. Achanta, A. Lucchi, P. Fua, A. shaji. Comparision of State-of-the-Art Superpixel Methods With SLIC Superpixels. IEEE Transactions on Machine Intellegenece and Pattern Analysis. 2012; 34: 2274-2282.

[9]. Li. Zhenhui and Han Jiawei., Gu.Quanquan,. Linear Discriminant Dimensionality Reduction, In Proc. of the 21st European Conference on Machine Learning (ECML), Athens, Greece. 2011.

[10]. K Sivakumar, J.Goutsias, H. Heijmans . Morphological operators for image sequences, Computer Vision and Image Understanding. 1995; 62: 326-346.

[11]. P. Mehrani, Boykov Y., O. Veksler. An energy optimization framework of Superpixels and supervoxels, in Proc. Europian Conference on Computer Vision. 2010; 6315: 211-224.

[12]. https://www.vlfeat.org/api/slic.html

[13]. https://towardsdatascience.com/an-illustrativeintroduction-to-fishers-linear-discriminant9484efee15ac.

[14]. C. Sabanayagam, R. Lee, and T. Y. Wong. Epidemiology diabetic macular edema, related vision loss and diabetic retinopathy, Eye and Vision. 2015; 2: 17 . 
[15]. T. Kauppi DIARETDB1 diabetic retinopathy database and evaluation protocol, https://www.it.lut.fi/project/imageret/diaretdb1/.

[16]. X. Zhang, Mass screening of diabetic retinopathy for exudates detection in retinal images for. Medical Image Anal. 2014; 18: 1026-1043.

[17]. G. Cazuguel, E. Decencier, X. Zhang. Feedback on a publicly available image database. Database Image Analysis Stereology of Messidor database. 2014; 33: 231-234. 